\title{
Steps through the Revision Process of Reproductive Health Sections of ICD-11
}

\author{
Doris Chou Özge Tunçalp Selen Hotamisligil Jane Norman Lale Say \\ On behalf of Genito-Urinary Reproductive Medicine TAG ${ }^{1}$
}

\begin{abstract}
In 2007, the WHO initiated an organizational structure for the 11 th revision of the International Classification of Diseases (ICD). Effective deployment of ICD-derived tools facilitates the use and collection of health information in a variety of resource settings, promoting quantitatively informed decisions. They also facilitate comparison of disease incidence and outcomes between different countries and different health care systems around the world. The Department of Reproductive Health and Research (RHR) coordinates the revision of chapters 14 (diseases of the genitourinary system), 15 (pregnancy, childbirth, and puerperium), and 16 (conditions originating in the perinatal period). RHR convened a technical advisory group (TAG), the Genito-Urinary Reproductive Medicine (GURM) TAG, for the ICD revision. The TAG's work reflects the collective understanding of sexual and reproductive health and is now available for review within the ICD-11 revision process.

Copyright $\odot 2012$ S. Karger AG, Basel
\end{abstract}

\section{Background}

The International Classification of Diseases (ICD) is a key instrument of the World Health Organization (WHO). The ICD is a member of the WHO Family of International Classifications (WHO-FIC) which seeks to provide a public global standard to organize and classify information about diseases and related health problems.
Initially adopted for coding causes of death in 1948, it has been revised approximately every 10 years, with the exception of the 20 years between ICD-9 and ICD-10 [1].

The WHO Nomenclature Regulations adopted in May 1967 during the 20th World Health Assembly emphasized the WHO's leadership in health information and the role of the ICD by stipulating the use of the most upto-date ICD version for mortality and morbidity reporting in all Member States [1].

Despite the original intent of the ICD, some consider the ICD only in the context of reimbursement. However, the ICD is a useful health information standard for coding morbidity, as well as for recording specific diseases, injuries, signs, symptoms, complaints, social circumstances, reasons for presentation, and external causes of both injury and disease. The ICD informs public health bodies, clinicians, and researchers alike in the environ-

1 On behalf of Genito-Urinary Reproductive Medicine TAG: Thomas D'Hooghe, Jacques de Mouzon, Jason Gardosi, Selen Hotamisligil, Mark A. Morgan, Jane Norman, Robert Pattinson, Cleone Rooney, Gamal Serour, Joachim Thüroff, Björn Volkmer.

- World Health Organization (2012). All rights reserved. The World Health Organization has granted the Publisher permission for reproduction of this article.

Disclaimer: The author is a staff member of the World Health Organization. The author alone is responsible for the views expressed in this publication and they do not necessarily represent the views, decisions or policies of the World Health Organization.

\section{KARGER}

Fax +4161306 1234

E-Mail karger@karger.ch

www.karger.com (c) 2012 S. Karger AG, Basel

$0378-7346 / 12 / 0743-0228 \$ 38.00 / 0$

Accessible online at: www.karger.com/goi
Dr. Doris Chou

WHO

20, Avenue Appia

CH-2700 Geneva (Switzerland)

E-Mail choud@who.int 
ment of increasingly complex health systems, ensuring the provision of standard and comparable definitions that are applied for:

- national and international health statistics (mortality and morbidity);

- epidemiology, surveillance, and monitoring;

- individual patient records and electronic health records;

- reimbursement and health system financing;

- reference for treatment guidelines, scientific literature, and research;

- quality assessment at the level of individual cases up to assessment of health system outcomes and monitoring.

Effective deployment of ICD-derived tools facilitates the use and collection of health information in a variety of resource settings, promoting quantitatively informed decisions. They also facilitate comparison of disease incidence and outcomes between different countries and different health care systems around the world.

As mentioned above, the ICD has generally been revised approximately every 10 years. ICD-10 was completed in 1990 and the World Health Assembly requested that it be revised as necessary, with such revision being organized and coordinated by the WHO Secretariat to provide support for the eventual transition from ICD-10 to ICD-11. It is important to note that ICD-11 is designed to be backwards compatible with ICD-10, in that explicit 'mapping' will be created to ensure that in the transition between ICD-10 and ICD-11 terminology and data will be comparable.

The aim of this article is to describe the process and methodology being used to revise the reproductive health sections (chapters 14,15, and 16) in the context of the revision process towards ICD-11.

\section{ICD-11 Revision Methodology and Progress to Date}

In 2007, the WHO initiated an organizational structure for the 11th revision of the classification. In contrast to previous revisions, this revision was intended to be internet based and open to the public in a structured way with mechanisms for information gathering, integration, and sharing. The WHO Secretariat coordinates the transition from ICD-10 to ICD-11 with the goals of: (1) the ICD evolving to become a multipurpose and coherent classification, establishing use in mortality, morbidity, primary care, clinical care, research, and public health surveillance contexts, (2) ensuring consistency and interoperability

\section{Box 1: What is the Content Model?}

The Content Model is a structured framework that defines 'a classification unit' in ICD in terms of its components. A 'model' is a technical term that refers to a systematic representation of knowledge that underpins any system or structure.

The components of the content model include the following parameters: titles, classification properties, definitions, terms, body structures, temporality, and severity [1].

Additional information on the content model can be found at http://tinyurl.com/icdrefguide-2011-01.

across different uses, and (3) serving as an international and multilingual reference standard for scientific comparability and communication purposes in an electronic environment [ICD Secretariat, pers. commun.]. ICD-11 is intended for use in all resource settings and will support electronic health records and information systems through linking ICD concepts logically to other classifications such as SNOMED and Gene Ontology.

An organizational structure was created to assist the WHO in the oversight and coordination of the revision process led by a revision steering group (RSG). RSG membership includes the chairs of individual Topic Advisory Groups (TAGs). The TAGs are organized to coordinate the revision of the ICD in specified domains. Overall, there are over 460 experts in over 26 TAGs (fig. 1). In large part, members of the TAGs donate their time for this work and, where possible, donors support this work.

To understand the revision process better, it is important to acknowledge the ICD-11 structure. ICD-11 is made up of entities constructed on a modern software platform, where each entity is defined to standardize interpretation by different users globally and facilitate implementation in electronic formats. Building from these entities, ICD-11 is a collection of multiple layers or 'use cases'. An apt analogy would be a multilevel cake (ICD11) which has different flavors (use cases), each created using different ingredients (content). More specifically, the goal is to formally represent the content in a systematic way, so that ICD-11 can be visualized in its entirety or as a 'sum of the various parts'. To improve understanding of the ICD-11 structure, the WHO published a reference guide on the structure of ICD-11 [2]. In addition, a computer platform, the international Collaborative $\mathrm{Au}$ thoring Tool (iCAT), was designed to allow TAGs to complete the details necessary to define the core content of 


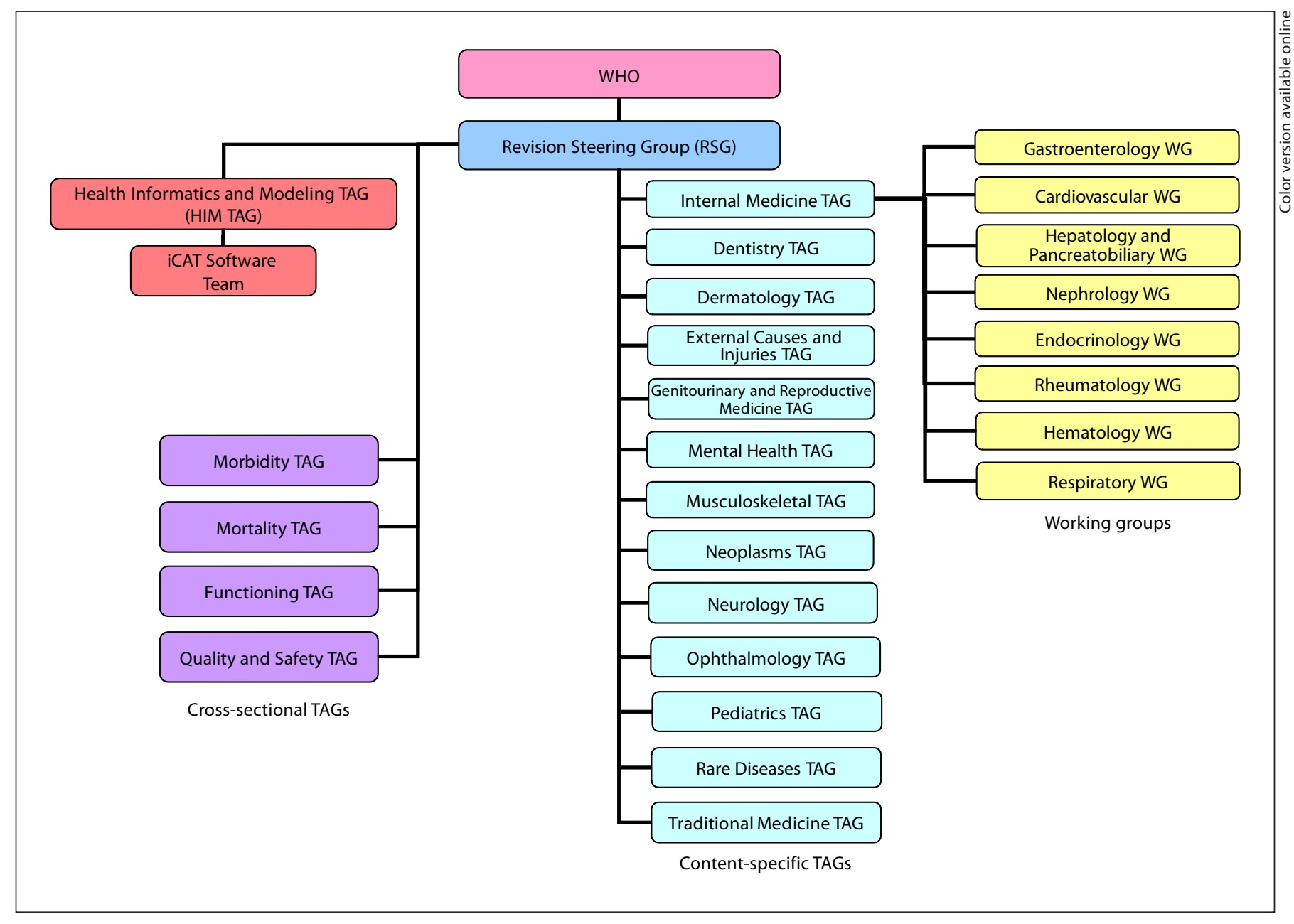

Fig. 1. Schematic diagram of the ICD revision organizational structure.

ICD-11 $[3,4]$. This content model is explained in box 1 . It should be noted that in comparison to the previous edition of the ICD, which did not provide explicit definitions for each entity/category, definitions are at the core of ICD-11. Contributions to the content of ICD-11 ideally are referenced by citation.

Eventually, entities from the content model can be reorganized into 'linearizations', which are specialized lists for different audiences and/or users. An example would be a primary care linearization. Within that linearization, concepts related to sexual and reproductive health may be described in the broadest terms such as 'urinary incontinence, female' but in a linearization created for urologists and urogynecologists, the concept may be further disaggregated to include all specific entities which might fall under such a heading. Linearizations do not modify the ICD; rather, they represent customized thematic lists much like a musical playlist represents a person's favorite songs for a particular occasion. In ICD-11, the most specific terms are meant to map back the broadest term, thus simplifying data comparability.

\section{Revisions Related to Sexual and Reproductive Health}

The Department of Reproductive Health and Research (RHR) coordinates the revision of chapters 14 (diseases of the genitourinary system), 15 (pregnancy, childbirth, and puerperium), and 16 (conditions originating in the perinatal period). In ICD-10, these chapters encompass over 1,400 clinical concepts. This coordination role includes 


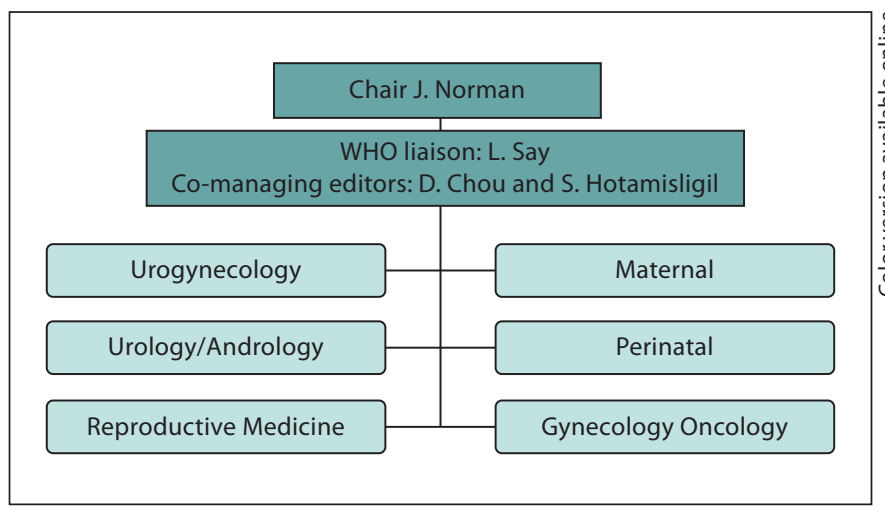

Fig. 2. Structure of the technical advisory group.

advising on the ICD-11 revision steps, establishing a technical advisory group made of six working groups (fig. 2), developing the various drafts of content for ICD-11 in line with the overall ICD-11 revision process, and identifying key partners and stakeholders for their involvement.

The first meeting of the Genito-Urinary Reproductive Medicine Technical Advisory Group (GURM TAG) for the ICD revision took place in October 2010 in Geneva, Switzerland. The aim was to introduce TAG members to each other and to devise a work plan for the revision process. Chapters 14, 15, and 16 were reviewed and a lead working group was designated for each category. The working groups were asked to consider core issues they would address for each diagnostic entity in their content area, and to develop a preliminary proposal on each issue based on the pre-existing knowledge of this domain. The group also established methods to discuss ownership within TAG and outside of TAG, and possible overlaps with other ICD TAGs were identified.

It was agreed that the lead working group (as identified during the meeting) would be responsible for completing the core content model for each entity. Any other overlapping working groups within this TAG would then be given the opportunity to comment on the proposals of the lead working group. For ICD categories owned by other TAGs but of interest to this TAG, the TAG chair and the WHO Secretariat were asked to inform the other relevant TAGs and to open discussions.

The GURM TAG continues its collaborative work over scheduled teleconferences and with email correspondence. In late 2012, the TAG will begin to review coding rules in volume 2 of the ICD and manage issues of maintaining congruence between the work of the GURM and other TAGs.

\section{Box 2: How to contribute to the ICD-11 beta phase}

Beta phase participants will have the opportunity to make comments, propose definitions of diseases in a structured way, participate in field trials, and assist in translating the ICD into other languages.

Step 1 - Go to the following URL:

http://apps.who.int/classifications/icd11/browse/f/en.

Step 2 - Log in to the browser (you can log in via thirdparty e-mail accounts such as Gmail).

Step 3 - After logging in, fill in the brief user profile.

Step 4 - You are now in the system and you can explore the site and comment!

\section{Progress to Date}

In May 2011, all elements of the core content model under the GURM TAG's responsibility were completed and agreed upon. By May 2012, 975 definitions had been completed, agreed upon, and entered into the iCAT authoring tool. During this process, collaborations with other TAGs such as mental health, dermatology, pediatrics, nephrology, oncology, and endocrinology were established and continue. As a result of the revision process thus far, the number of identified clinical concepts has also increased to over 2,600.

As this revision is envisioned as a collaborative exercise, over 180 professional organizations around the world have been invited to comment on the ICD along with step-by-step instructions. To further share the process and the experiences, a special session at the International Federation of Gynecologists and Obstetricians (FIGO) meeting in October 2012 is designated to discuss the ICD-11 revision as it relates to the reproductive health sections. Included topics are perspectives on pitfalls of the ICD and vital registration from Jamaica, the ICD-10 maternal mortality application, and a discussion of the ICD-11 process and what can be done to make it suitable for all users.

As of July 2012, the ICD revision process is in the beta phase. The ICD Secretariat has opened the platform to the public so that all interested stakeholders are able to comment. During this commenting phase, further revisions to ICD-11 will be managed by the ICD Secretariat. Additionally, plans for field trials of ICD-11 will be made in the near future. 


\section{Conclusions}

ICD-11 is scheduled for release in 2015; however, implementation by countries will require widespread systematic and programmatic changes. Thus, to ensure data comparability, until such efforts are completed the WHO will create specific 'translators' of ICD-11 to match the ICD-10 entities, thereby ensuring backwards compatibility with previous version of the ICD.

The GURM TAG has successfully completed draft definitions for the majority of entities in chapters 14, 15, and 16. To ensure that the efforts of the GURM TAG are complete and reflect the collective understanding of sexual and reproductive health, the TAG has tried to contact specialist society/national groups who might have an interest and ask for their comments directly. The work of the TAG will also be presented at FIGO 2012 to invite a broader contribution.

Currently the process is open to the public and scientific community for comment, and the details of how to do so can be found in box 2 .

The path towards revising the ICD has been fraught with challenges. At first glance, the revision process can be overwhelming and maintaining the balance of detail for each entity, within each working group, TAG, and between TAGs, requires significant communication and technical effort on the part of the ICD Secretariat. The
GURM TAG has endeavored to complete the core content model to represent advancements in scientific knowledge related to sexual and reproductive health. The GURM TAG has been fortunate to collaborate with several other TAGs, capitalizing on shared expertise to complete the core content model. Finally, the GURM TAG is acutely aware of the importance of this work, as some proposed changes may impact monitoring and evaluation of progress towards and beyond the Millennium Development Goals. The specific attention given to ensuring ICD-11 applicability in low-resource and developing countries is vital to answering calls for improvements in vital registration which require improved understanding and appropriate use of ICD codes [5]. The inclusion of definitions in ICD-11 will aid interpretation and improve data quality for international comparability.

\section{Acknowledgements}

The GURM TAG is grateful to the WHO ICD Secretariat for their support in the revision process. The authors acknowledge Drs. Robert Jakob and Molly Meri Robinson for their review and comments on the manuscript.

This work was funded by USAID and the Department of Reproductive Health and Research, WHO, through the Special Programme of Research, Development, and Research Training in Human Reproduction.
References
1 World Health Organization: International Classification of Diseases (ICD). Geneva, WHO, 2012.

2 ICD-11 Alpha: Content Model Reference Guide, ed 11. Geneva, WHO, 2010.

3 International Classification of Diseases: ICD Collaborative Authoring Tool. 2012.
4 Tu W, Bodenreider O, Celik C, Chute CG, et al: A Content Model for the ICD-11 Revision: Technical Report 2010. http://bmir.stanford. edu/publications/view.php/a_content model_for_the_icd_11_revision.

5 Keeping promises, measuring results: Commission on Information and Accountability for Women's and Children's Health. Geneva, WHO, 2011. 


\section{Erratum}

In the article by Chou et al: Steps through the Revision Process of Reproductive Health Sections of ICD-11. Gynecol Obstet Invest 2012;74:228-232, please add the following names to the list of authors: Björn Volkmer, Bob Pattinson, Cleo Rooney, Gamal Serour, Jacques de Mouzon, Jason Gardosi, Joachim Thueroff, Morgan Mark, Thomas D’Hooghe. 